\title{
GLAD!
}

Revue sur le langage, le genre, les sexualités

11 | 2021

Archives, genre, sexualités, discours

\section{Les affiches de la lutte contre le sida}

Archives oubliées de la mobilisation gaie à Montréal (1982-2012)

Posters of the Fight against AIDS. Forgotten Archives of the Gay Mobilization in

Montreal (1982-2012)

\section{Alexandre Klein et Gabriel Girard}

\section{(2) OpenEdition}

Journals

Édition électronique

URL : https://journals.openedition.org/glad/2942

DOI : $10.4000 /$ glad.2942

ISSN : 2551-0819

Éditeur

Association GSL

\section{Référence électronique}

Alexandre Klein et Gabriel Girard, « Les affiches de la lutte contre le sida », GLAD! [En ligne], 11 | 2021,

mis en ligne le 20 décembre 2021, consulté le 29 janvier 2022. URL : http://journals.openedition.org/ glad/2942 ; DOl : https://doi.org/10.4000/glad.2942

Ce document a été généré automatiquement le 29 janvier 2022.

\section{c) (†)}

La revue GLAD! est mise à disposition selon les termes de la Licence Creative Commons Attribution -

Pas d'Utilisation Commerciale - Pas de Modification 4.0 International. 


\title{
Les affiches de la lutte contre le sida
}

\author{
Archives oubliées de la mobilisation gaie à Montréal (1982-2012) \\ Posters of the Fight against AIDS. Forgotten Archives of the Gay Mobilization in \\ Montreal (1982-2012)
}

Alexandre Klein et Gabriel Girard

1 À notre arrivée, au début de l'été 2019, dans l'ancien presbytère qui accueille désormais les bureaux de l'organisme communautaire REZO, aux abords du village gai à Montréal, notre impatience était à son comble. Suite à nos différentes requêtes concernant leurs archives, on nous avait laissés entendre qu'une salle entière était réservée aux souvenirs des mobilisations, parmi lesquels des affiches de la lutte contre le sida qui pourraient nous intéresser. Et quelle ne fut pas, en effet, notre surprise! Au milieu d'une grande pièce aux murs rose saumon trônait un imposant amoncellement d'objets divers, posés à même le sol ou entreposés sur des bureaux, des tables et des chaises également stockés là. Des ballons d'exercice, des tas de rallonges électriques emmêlées, un vélo, une épuisette rose et même un grand sapin de Noël artificiel émergeaient aux côtés de boites remplies de tracts, de livres ou de documents divers, de vieilles photographies encadrées, d'anciennes pancartes, ainsi que de banderoles et de drapeaux de la fierté. Au cœur de cet amoncellement bigarré, des tubes cartonnés dépassant d'une grande poubelle grise nous laissaient entrevoir ce pourquoi nous étions là. Des dizaines d'affiches de la lutte contre le sida, de différentes époques et origines, en français, en anglais ou plus rarement en espagnol, gisaient là, attendant simplement qu'on vienne les (re)découvrir. Véritable cauchemar pour un archiviste, cette caverne d'Ali Baba communautaire, qui fit rapidement notre bonheur, témoignait d'années de militantisme actif, où l'urgence laisse peu de place à l'archivage et à l'écriture de l'histoire en train de se faire. Elle se faisait aussi l'écho d'un enjeu de taille pour cet organisme alors en cours de réorganisation de son espace de travail (cette salle aux trésors devait en effet rapidement laisser place à de nouveaux espaces de travail) : que faire de tous ces documents autour desquels s'organisent les luttes, grâce auxquels elles prennent corps? Faut-il tous les conserver, et si oui comment? Faut-il n'en conserver qu'une partie, mais dès lors laquelle? Et surtout où stocker, dans de bonnes 
conditions, ces archives qui ne sont plus directement utiles à la vie de l'organisme, alors même que les espaces tout comme les financements manquent ?

2 Ces questions, de nombreu-ses militant·es se les sont posées, et ce depuis des décennies. Et différentes initiatives ont vu le jour pour tenter d'y répondre et d'ainsi sauvegarder au mieux les traces des luttes et des engagements. Dès les années 1970, les mouvements que l'on nomme aujourd'hui LGBTQIA+ avaient pris les devants pour sauvegarder leurs mémoires. Ainsi, en 1973 furent fondées les Canadian Gay Liberation Movement Archives (aujourd'hui les ArQuives qui se revendiquent comme le plus important centre d'archives LGBTQ2+ indépendant au monde ${ }^{1}$ ), puis, dix ans plus tard, les Archives Gaies du Québec (AGQ) qui se donnèrent pour mandat de conserver et préserver les traces de l'histoire des communautés gaies (et aujourd'hui LGBTQ+) de la province ${ }^{2}$. La France rejoint aujourd'hui, avec un certain retard, ce mouvement international puisque le 2 février 2021, après plusieurs décennies d'atermoiements, et sous la pression des activistes ${ }^{3}$, le conseil de Paris a adopté « le principe de la création d'un lieu "autonome" destiné à conserver, à valoriser et à faire vivre la mémoire des minorités sexuelles ${ }^{4}$. Entre-temps, l'épidémie de VIH-sida avait renouvelé le besoin de préservation des mémoires et des luttes. Elle a notamment conduit à la création de collections dédiées dans les lieux d'archives déjà existants, comme ce fut le cas aux AGQ où furent sauvegardées des archives privées de personnes emportées par l'épidémie ou avec le collectif Visual AIDS 5 , fondé en 1988 aux États-Unis pour valoriser le rôle de l'art dans la lutte contre l'épidémie mais aussi en sauvegarder les traces. Elle a également mené à la formation de fonds propres ensuite déposés dans des institutions de conservation plus traditionnelles, comme ce fut le cas avec le dépôt, dès 1999, des archives d'AIDES aux Archives nationales de France, puis avec celles d'Act Up-Paris en 2014, ou encore avec la création à New York aux débuts des années 2000 de l'Act Up Oral History Project ${ }^{6}$ en lien avec la bibliothèque de l'Université Harvard. A Marseille, la collection « VIH/sida » du Musée des civilisations de l'Europe et de la Méditerranée (Mucem) témoigne également d'une démarche ancienne et active de problématisation ${ }^{7}$, de collecte et de valorisation d'archives très diverses de ce mouvement social (banderoles, tracts, photographies, etc.) . $^{8}$

3 Cependant, ce souci de l'archive demeure, sur la longue durée, une démarche minoritaire, pas toujours connue et souvent portée par une poignée d'individus bénévoles passionnés et sensibles à ces enjeux. Dans le cas de REZO, par exemple, si par le passé, des membres (actuels ou anciens) ont pu faire don de nombreux documents aux $\mathrm{AGQ}$, la question de l'archivage du temps présent n'est logiquement pas une priorité pour l'organisme, et ce d'autant plus que les outils/visuels/campagnes de lutte contre le VIH sont de plus en plus numériques.

4 Les intérêts et les temporalités des militant-es ne sont en effet pas nécessairement ceux des historien-nes et des archivistes. Les premieres sont souvent prises dans l'urgence du quotidien ou le temps court des prochaines mobilisations, tandis que les secondes pensent généralement dans le temps long des sociétés et de leurs transformations, tentant de percevoir à distance ce qui a pu se jouer dans des gestes répétés ou des évènements singuliers. Parfois, heureusement, ilselles parviennent à se rencontrer, à se parler, à mutualiser leurs démarches et leurs efforts ${ }^{10}$, mais cela reste encore trop rare. Et surtout cela n'empêche pas que les unes et les autres portent des regards différents sur les traces des luttes et en particulier sur les affiches qui n'ont à leurs yeux ni la même valeur, ni la même signification. 


\section{Archives et sources à part entière}

Pour celles et ceux qui les produisent, les collent ou les distribuent, les affiches ne sont en effet que le support le plus souvent éphémère (car « daté » ou « situé » dans l'espace et le temps) et infiniment reproductible d'un message à passer qu'elles ne font que matérialiser. Pour celles et ceux qui tentent de comprendre, rétrospectivement, les évènements, les gestes et les postures liés à cette épidémie, elles sont au contraire des traces précieuses, des sources parfois devenues rares, en tout cas des témoins incontournables des évènements passés. Ou du moins le sont-elles devenues. Car malgré leur omniprésence dans le champ militant - et les efforts de valorisation dont elles peuvent faire l'objet ${ }^{11}$ - les affiches n'ont que tardivement intéressé les chercheur.es, et restent encore l'objet d'un intérêt très restreint ${ }^{12}$, même de la part de celleux qui ont travaillé sur les représentations médiatiques des malades et de la maladie $^{13}$. Pourtant, l'histoire de la lutte contre l'épidémie de VIH/sida a rapidement intéressé, dans différents pays à travers le monde, les sociologues d'abord, puis les historien'nes ensuite, mais sans qu'ils·elles prennent les affiches au sérieux. Situation d'autant plus paradoxale que ces dernières furent pourtant les symboles mêmes de la lutte contre l'épidémie ainsi qu'en témoigne la célèbre affiche Silence=Death, créée à la fin des années 1980 par un collectif éponyme et qui incarna à elle seule l'épidémie et ses enjeux politiques, notamment suite à sa reprise par Act Up - New York.

Certes, des projets récents, issus des histoires des mouvements militants, et notamment des mouvements LGBTQIA+ actuellement en pleine expansion, les ont remis au centre de l'intérêt historiographique. En France, le sociologue Antoine Idier leur a par exemple accordé une place importante dans sa somme intitulée Archives des mouvements LGBT+ : une histoire de luttes de 1890 à nos jours parue en 2018. Au Canada, le AIDS Activist History Project ${ }^{14}$ les inscrit également au cœur de sa démarche. Mais dans un cas comme dans l'autre, les affiches, bien qu'archives à part entière, ne sont pas réellement utilisées comme des sources pour écrire une histoire, et notamment une histoire de la lutte contre le sida. Elles apparaissent bien plus comme des artefacts mobilisés, certes pour leur valeur historique, mais aussi et surtout pour leur valeur esthétique et leur portée politique. Bref, les affiches n'y sont pas réellement envisagées comme des objets d'étude à part entière, alors même que leur potentiel historiographique apparait immense, ainsi qu'en témoignent d'autres démarches de compilation d'affiches de santé publique au Québec ${ }^{15}$ ou en France ${ }^{16}$.

7 Les affiches sont en effet des sources de choix pour écrire l'histoire de l'épidémie de $\mathrm{VIH} /$ sida. D'abord, parce qu'elles furent au cœur même de la lutte contre le sida comme le rappellent les expositions Sidart ou Visual AIDS établies en parallèle de la Conférence internationale sur le sida à Montréal de juin 1989 et qui mettaient en vedette les affiches dont nombreuses avaient été créées spécifiquement pour l'occasion ${ }^{17}$. Ensuite, parce qu'elles révèlent les engagements des uns et des autres, leurs positionnements distincts, leurs stratégies, leurs réseaux d'influence (et en particulier la capacité à mobiliser des créateurs ou des artistes pour certaines affiches), mais aussi leurs cibles prioritaires ou leur langue ${ }^{18}$. Bref, elles donnent à voir, si on leur accorde leur légitime statut de sources historiques à part entière, l'organisation même de la lutte contre le VIH et son évolution au fil du temps, dessinant ainsi une carte virtuelle de ses acteurs et actrices et de leurs champs d'expertise et donc d'exercice. Ces affiches sont par 
ailleurs des témoins passionnants du processus de traduction et d'appropriation - par les acteurs communautaires, institutionnels ou médicaux - des recommandations (et des catégories) de la santé publique sous forme visuelle. Elles ont donc toute leur place dans l'écriture de l'histoire de la lutte contre le sida, ainsi que l'avait déjà laissé entrevoir l'exposition Si le sida m'était conté. Images d'une pandémie, organisée en 2001 à l'Écomusée du fier monde à Montréal en partenariat avec les AGQ. C'est aussi le constat que nous avons pu faire en débutant notre étude sur les affiches de la lutte contre le sida à Montréal entre 1982 et $2012^{19}$.

\section{Cartographie montréalaise}

8 Les multiples affiches que nous avons pu retrouver (plus de 500), que ce soit en dépouillant d'abord la riche collection des Archives Gaies du Québec (et notamment le fonds Ken Morrison incluant les affiches de l'exposition Sidart), ou en collectant ensuite, dans divers organismes communautaires, les affiches de leurs mobilisations passées, témoignent en effet des différentes stratégies des acteurs et actrices impliquées dans la prévention, mais également de la variété de leur cible et de leur stratégie pour les atteindre. Face à un gouvernement provincial frileux à l'égard des questions d'homosexualité et qui tarde donc à se mobiliser, les groupes communautaires gais apparaissent comme les moteurs d'une lutte contre l'épidémie qui se veut aussi l'occasion de revendications sociales et politiques identitaires fortes. Tout en confirmant ce que nous avaient laissé comprendre les rares écrits sur l'histoire montréalaise du sida ${ }^{20}$, et les quelques entrevues que nous avons réalisées avec des acteurs et actrices de l'époque, ces affiches dessinent une cartographie inédite et particulièrement éclairante des différents acteurs institutionnels engagés dans la lutte contre le sida depuis son apparition dans la province canadienne en 1982 et de leurs rôles propres.

9 On y aperçoit clairement le rôle central et précoce du Comité Sida Aide Montréal (CSAM), créé en 1983 par des membres de la - toute nouvelle, à l'époque - Association des ressources montréalaises sur le SIDA (mais financé par le gouvernement québécois). Ses premières affiches sont simples, informatives, visant à expliciter son rôle: informer, assister, intervenir. Puis viennent les affiches plus axées sur la prévention comme la fameuse campagne Jouez sûr/Play safe, lancée en $1985^{21}$ pour la promotion du préservatif - la première au Québec à viser explicitement la communauté gaie - et qui montre des mains sur un torse tenant une ribambelle de condoms. 
Affiche de la campagne Play Safe/Jouez sûr, CSAM, 1985, Archives gaies du Québec (Photographie A.Klein)

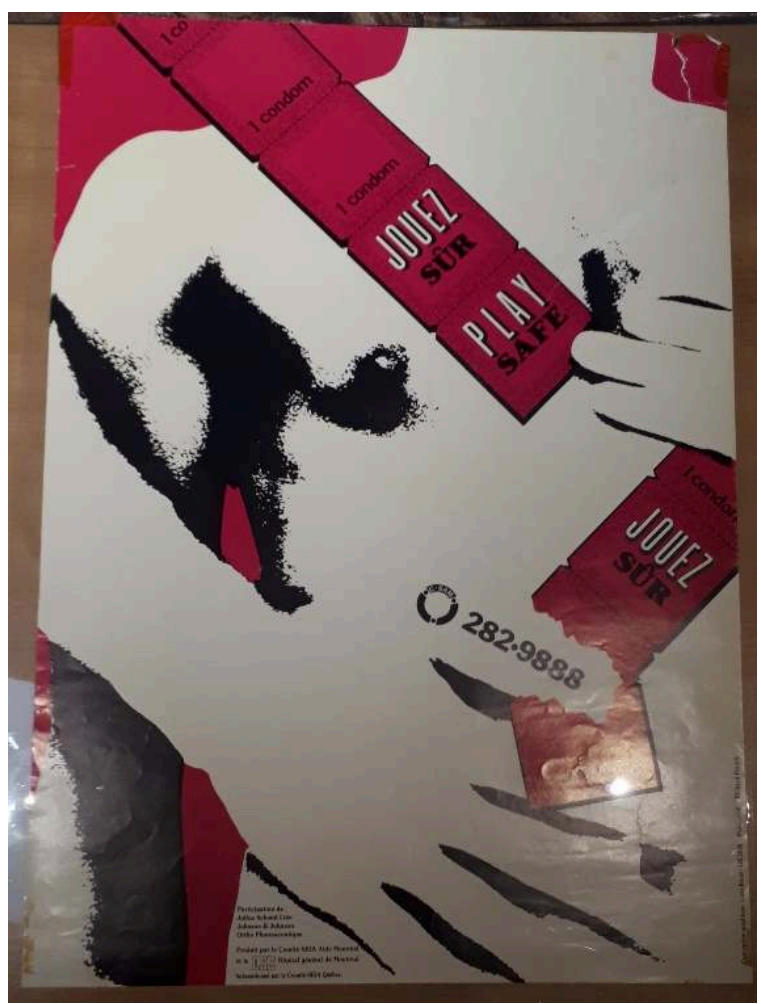

D'autres campagnes seront ensuite plus suggestives, mettant en scène et en photographie des corps nus et des sexes en érection, comme la campagne Le plaisir du Latex. Mais, dans l'ensemble, les affiches du CSAM retrouvées restent sobres, colorées, informatives, voire joviales, à l'image des visuels pour leurs ateliers $4 \mathrm{~S}$ (sexe, sensuel, stimulant, sécuritaire). Bref, des affiches orientées " grand public » en un sens, même si elles s'adressent d'abord et avant tout aux communautés d'hommes homosexuels québécois, francophones comme anglophones. Il s'agit alors d'informer sans stigmatiser. 
Affiche de la campagne Sécurisexe, CSAM, c. 1990, Archives gaies du Québec

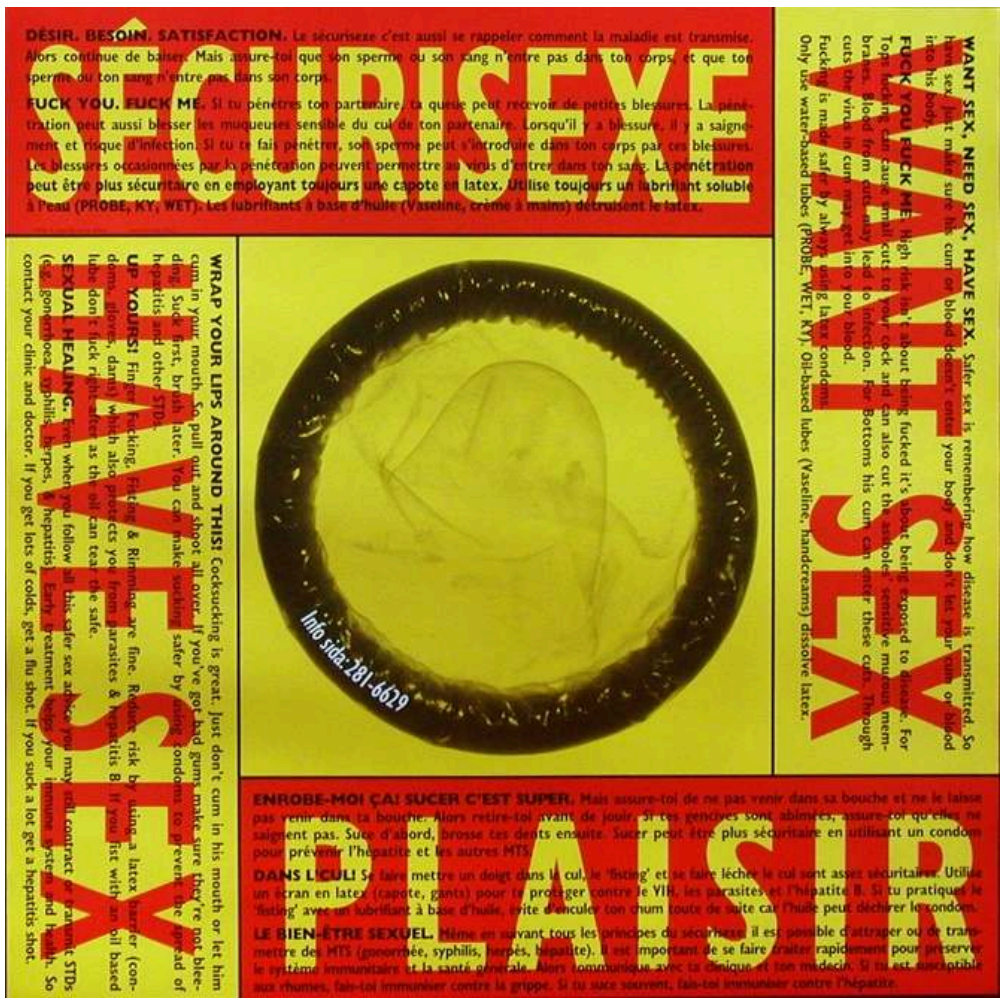

11 La deuxième association qui émerge du lot des affiches consultées est Act Up Montréal. À l'inverse des couleurs vives du CSAM, ses affiches sont généralement en noir et blanc, le ton y est grave et les slogans accusateurs. Créée à l'image de l'association newyorkaise, et dans la foulée de la conférence internationale sur le sida de 1989 qui avait été marquée par les revendications des associations de malades ${ }^{22}$, Act Up Montréal se place sur un crédo d'emblée plus politique. Ses affiches visent d'ailleurs souvent directement les gouvernants comme le premier ministre québécois Robert Bourassa (1985-1994) ou son ministre de la Santé Marc-Yvan Côté (1989-1994), tous deux membres du Parti Libéral du Québec (centre-droit). L'une d'elles reprend même le drapeau de la province pour mieux dénoncer, à coup de phrases-chocs, l'inaction du gouvernement. 
Affiche d'Act Up Montréal, 1992, Archives gaies du Québec (Photographie A.Klein)

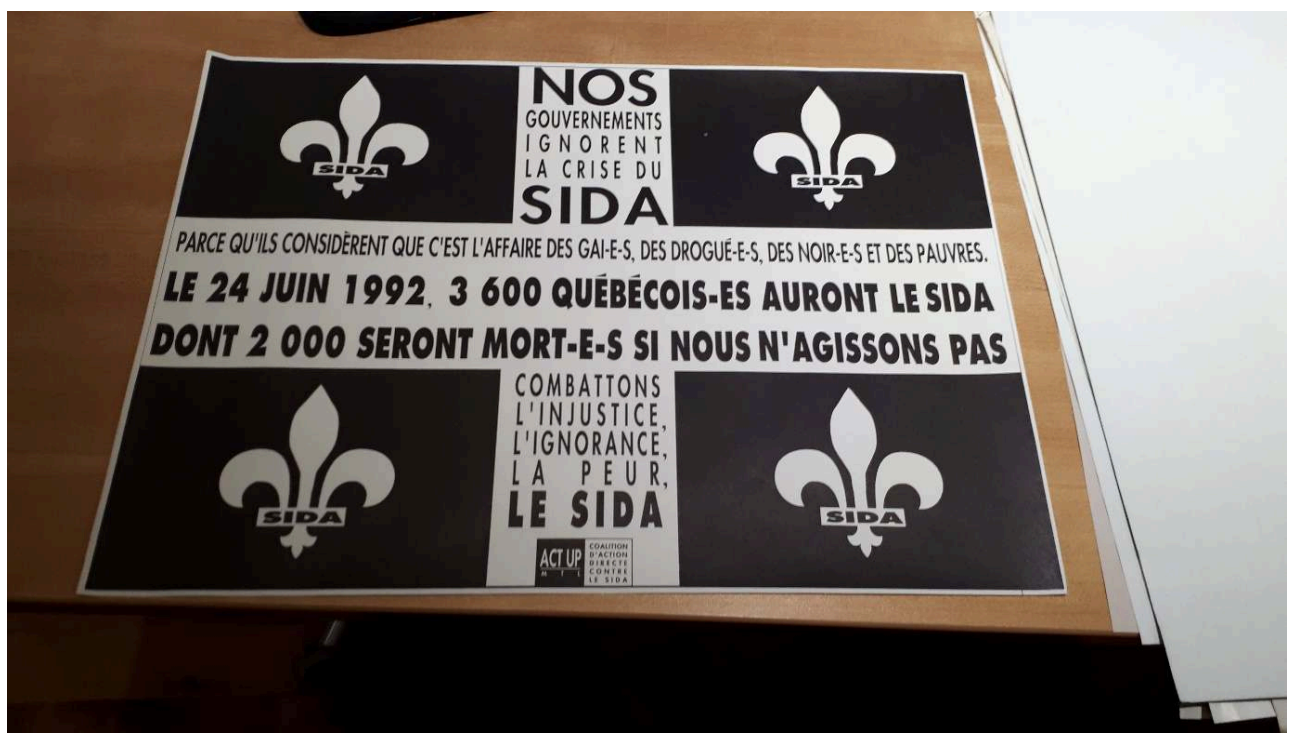

Le propos est engagé et plus radicalement politique que chez CSAM. Mais pas uniquement. Act Up Montréal produit aussi du matériel de prévention et d'information plus "classique», à destination de la communauté gaie et de ses lieux de rassemblement : bars, saunas, etc., ainsi qu'en témoignent les affiches retrouvées de sa campagne Safer Sex. À la différence de la communication des associations "généralistes ", Act Up Montréal cherche à s'adresser aux gais et aux lesbiennes à travers des dessins explicitant les pratiques sexuelles ou les pratiques de consommation de drogues.

13 Le troisième organisme important, à cheval peut-être entre les engagements politiques d'Act Up Montréal et la démarche d'abord informative et préventive du CSAM, est Action Séro-Zéro. Ce positionnement tiers n'a rien d'étonnant puisque l'organisme est le fruit d'un projet commun, qui voit le jour en $1990^{23}$, mené par des représentant·es de différentes associations québécoises de lutte contre le sida. Ses affiches sont axées sur la prévention et la sensibilisation des hommes gais aux risques du VIH, mais aussi sur la création d'espaces d'échanges et d'informations, sans négliger les enjeux d'inclusion et de lutte contre la sérophobie. L'usage du préservatif est particulièrement présent dans les différentes affiches, depuis celles des années 1990, jusqu'aux plus récentes émises à l'occasion des Outgames de Montréal en $2006^{24}$. La multiplicité des affiches retrouvées laisse également entrevoir l'évolution des stratégies de prévention, à mesure de l'avancée de l'épidémie et de l'apparition de nouveaux traitements, mais aussi celle des esthétiques tantôt jouant sur le kitsch, tantôt humoristique, tantôt expérimentale, tantôt visionnaire, tantôt déjà datée. 
Affiche "Le droit d'aimer sans peur et sans reproche ", Séro-Zéro, c. 1990, Archives gaies du Québec (Photographie A. Klein)

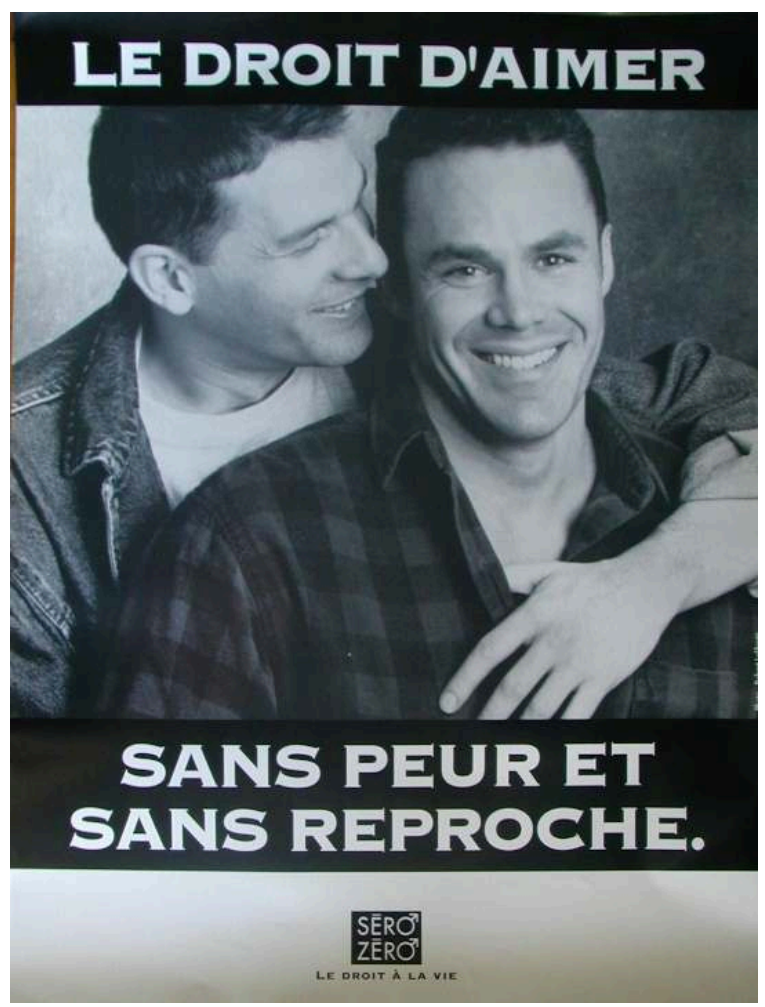

14 Les grands évènements publics favorisent les collaborations et on voit alors les organismes s'associer pour mutualiser leurs forces et majorer leur impact sur la population visée. Les affiches "sportives » invitant à l'usage des préservatifs lors des Outgames de 2006 sont ainsi co-signées par Séro-Zéro et la COCQ-Sida, une Coalition des organismes communautaires québécois de lutte contre le sida née en 1990 et dont les campagnes seront beaucoup tournées vers la prévention, la lutte contre les discriminations et la sérophobie. La campagne Pensez-y permet, elle, à Séro-Zéro de s'associer avec l'organisme AIDS Community Care Montreal, le seul organisme communautaire de langue anglaise au Québec offrant des services aux personnes vivant avec le VIH. La réunion des forces et l'entraide des organismes est monnaie courante comme en témoigne le Menu securisexe créé aux débuts des années 1990 par Séro-Zéro et qui présente des annonces pour le CSAM ou pour le CPAVIH, le Comité des personnes atteintes du VIH du Québec dont on trouve également quelques rares affiches dans les fonds explorés. Dans certains cas, les collaborations sont même internationales, à l'image de la campagne sijetaisseropositif.org initiée en 2010 pour laquelle la COCQ-Sida s'associe à l'association française AIDES. Dans certains cas néanmoins, les rôles sont bien répartis entre les organismes, notamment en ce qui concerne la prévention pour les usager·es de drogues injectables qui reste plus spécifiquement l'objet d'organismes dédiés comme l'Association des intervenants en toxicomanie du Québec.

Lociations nationales et internationales ne sont pas absentes de notre corpus : la Société canadienne du sida, l'Association canadienne de santé publique ou encore l'Organisation mondiale de la santé ont produit de nombreuses affiches, soit généralistes sur les modes de transmission du virus et la prévention, soit sur les enjeux de discriminations des personnes vivant avec le VIH, soit autour d'évènements nationaux ou internationaux particuliers, notamment les journées internationales du 
sida, qui ont lieu le 1er décembre à partir de 1988, ou en lien avec la Conférence internationale sur le sida qui se déroule à Montréal l'année suivante.

Le gouvernement provincial se mobilisa aussi, d'abord en finançant le CSAM nous l'avons vu, mais aussi en mettant en place en 1989, le Centre québécois de coordination sur le sida (CQCS) ${ }^{25}$ qui produira quelques affiches elles aussi assez généralistes, souvent tournées vers la jeunesse.

"Le sida, défi communautaire ", Centre québécois de coordination sur le sida, c. 1990, Archives gaies du Québec. (Photographie A.Klein)

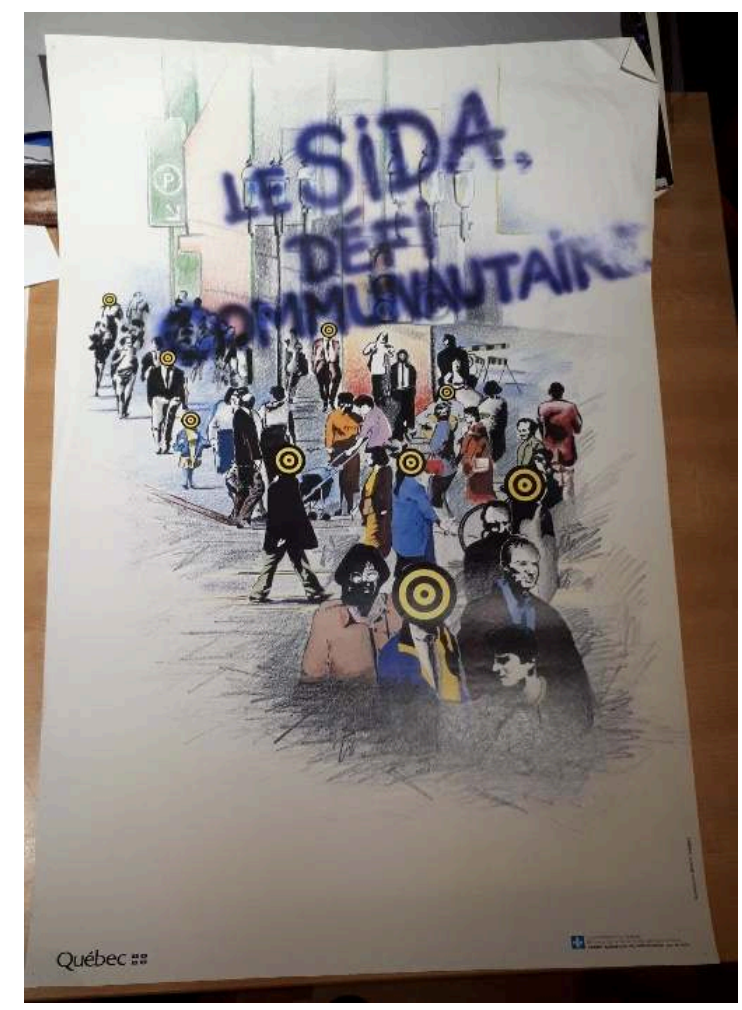

17 Même chose pour son ministère de la Santé dont on trouve plusieurs traces, mais pour des campagnes de prévention et d'information souvent à large spectre, c'est-à-dire visant rarement des populations spécifiques. La ville de Montréal aussi a produit au moins une affiche en 1988, pour inviter à l'usage du préservatif; nous n'avons pas retrouvé d'exemplaire de cette affiche mais nous avons pu retrouver des photographies de l'inauguration. Même chose : le propos est particulièrement sobre et consensuel, car le public visé est large. On comprend que les autorités, intervenues bien tardivement à la fin de la décennie 1980, préfèrent déléguer l'initiative aux groupements communautaires, plus à même, à leurs yeux, de communiquer avec les groupes minoritaires qu'elles représentent ou soutiennent. Elles se contentent donc d'interventions que l'on pourrait dire symboliques, parce que visant d'abord à montrer leur implication dans la lutte, favorisant plutôt le financement direct des organismes communautaires, notamment gais, pour qu'ils se chargent de mener les campagnes de lutte et de prévention auprès des populations concernées.

Enfin, on découvre aussi, au milieu de ces dizaines d'affiches dépouillées, des organismes moins connus, souvent occultés des grands récits historiques pour des raisons d'éloignement géographique avec Montréal, comme Miels Québec, le 
Mouvement d'information et d'entraide dans la lutte contre le sida à Québec créé en 1986 sous le nom de CIELS, ou parce que dédiés à un public très particulier comme la Coalition Sida des Sourds du Québec ou la Ligne info sida pour les sourds(es) et malentendants(es). Les affiches à l'attention des peuples autochtones du Québec sont aussi peu présentes, à la fois parce que ces communautés ont souvent leur propre réseau de prévention qui ne privilégie pas forcément les affiches comme moyen de communication, mais aussi parce que les associations qui les représentent sont moins nombreuses et moins actives au Québec que dans d'autres provinces canadiennes comme la Colombie-Britannique par exemple pour laquelle nous avons retrouvé plusieurs affiches explicitement dédiées aux Premières Nations. Au cours des années 1990, le Centre d'amitié autochtone de Montréal semble néanmoins être un acteur à part entière de la lutte contre le sida dans ces communautés. Même chose pour la communauté haïtienne montréalaise par exemple, pour laquelle nous n'avons pas trouvé d'affiches dédiées, mais dont on sait, notamment grâce au travail de Viviane Namaste $^{26}$, qu'elle a utilisé davantage les médias communautaires et en particulier les émissions de radio pour partager des informations de prévention face au VIH. Il faut admettre aussi que nos sources ne sont pas exhaustives tant parce que certains organismes communautaires locaux n'ont pas encore été sollicités, notamment les organismes haïtiens ou anglophones, que parce que certains ne semblent avoir laissé que de rares traces iconographiques, à l'instar de l'association militante Réaction $\operatorname{SIDA}^{27}$, qui fut pourtant à l'avant-plan lors de la mobilisation des associations de malades en ouverture de la Conférence internationale sur le sida de Montréal en 1989.

Affiche " Le silence = la mort », Réaction sida, c. 1990 (Aides Activism History Project)

\section{Réaction SIDA}
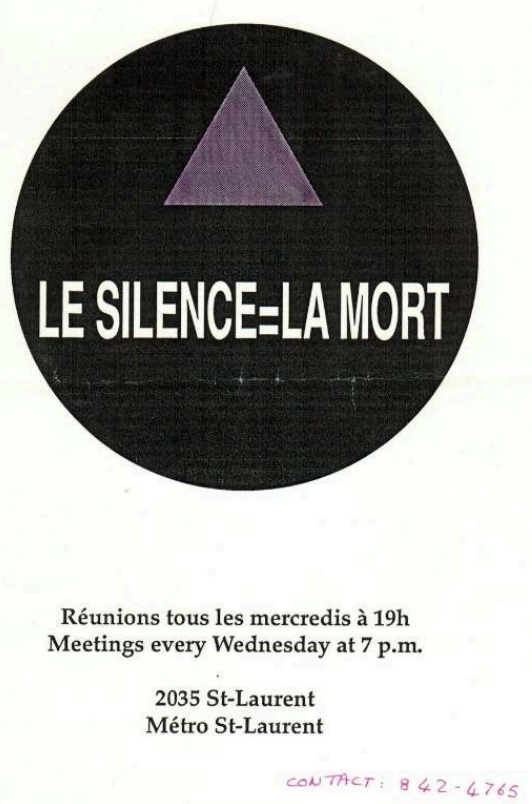


\section{Afficher la lutte : leçons et perspectives}

19 Ainsi, bien qu'elle soit encore en cours, notre recherche autour des affiches de la lutte contre le sida à Montréal entre 1982 et 2012 est déjà riche d'enseignements. D'une part, elle nous permet de mieux cerner les différents acteurs de la lutte et la répartition de leurs rôles et activités propres sur le territoire et à l'égard de la population québécoise comprise dans sa diversité. Elle nous invite ainsi à considérer Montréal comme un lieu à part entière de la lutte contre le sida, ainsi que le laissaient déjà entendre les évènements de la Conférence de 1989 ; un lieu périphérique certes par rapport à Paris, New York ou Toronto, mais néanmoins important, notamment du fait d'une communauté gaie ancienne, forte, et particulièrement active et résiliente face à l'épidémie. D'autre part, elle confirme le rôle central de l'affiche dans l'écriture d'une histoire du sida au Québec qui reste pour beaucoup à réaliser, mais aussi les importants défis qui s'attachent à sa considération comme source à part entière. Nous faisons ainsi face à un immense travail de datation des affiches. Car si certaines campagnes sont connues et bien identifiées, d'autres doivent, au contraire, faire l'objet d'importantes recherches pour en situer l'apparition et les conditions de création. Comme les organismes communautaires n'ont, en plus, pas toujours conservé les traces ou la mémoire de leurs campagnes passées, cette enquête risque d'être à la fois fastidieuse et en partie inévitablement infructueuse. Pour cette même raison, l'interprétation iconographique - discursive devrait-on dire - des affiches reste à ce jour l'une de nos principales difficultés. On s'interroge parfois sur les choix esthétiques, sur les influences, notamment étrangères, à l'œuvre dans certains choix de thématiques picturales, de messages ou de motifs; mais sans possibilité d'échanges avec leurs créateurs ou créatrices, le chemin risque d'être long et incertain. Pour pallier ce manque, nous combinons la méthode historique et sa classique double critique (interne et externe) à l'analyse linguistique, à la fois sémiotique et rhétorique, utilisée par Wang $^{28}$ afin de reconstituer le fil des évènements encadrant (et donc influençant) la production de ces différentes affiches, mais aussi de faire émerger les formes de discours à l'œuvre dans cette pratique de diffusion d'informations. La combinaison de ces deux approches nous permet de saisir les affiches à la fois pour ce qu'elles sont et dans le contexte spécifique et mouvant dans lequel elles ont été pensées puis produites. Elle nous invite notamment à être attentifs aux biais rétrospectifs et à ne pas juger les affiches à l'aune de connaissances scientifiques ou de réalités politiques postérieures à leur production. Ainsi, il apparait nettement que la période suivant l'introduction des multithérapies (1996-2000) a donné lieu, au Québec comme ailleurs, à une communication ciblant "l'optimisme thérapeutique » et l'abandon du préservatif supposé en découler. Au regard des connaissances actuelles sur le rôle préventif des traitements antirétroviraux, ces affiches apparaissent surprenantes, mais elles sont révélatrices d'une période d'incertitude stratégique au sein de la lutte contre le VIH. La mutualisation des approches méthodologiques nous conduit également à prendre en compte, au-delà de ce qui est nommé, ce qui est tu, afin de voir de quoi ces enjeux sont révélateurs socialement et politiquement. L'abondance d'affiches produites par et pour les communautés gaies donnent ainsi à voir une vision de la sexualité assumée et diverse, par les images comme par les textes. À l'inverse, les affiches concernant les femmes sont plus rares, souvent centrées sur la maternité ou sur le soin apporté aux autres - à l'exception notable des affiches militantes où les femmes vivant avec le VIH ont droit de cité ; quant aux hommes hétérosexuels, ils sont notoirement absents des 
discours et des représentations - à l'exception des publics jeunes et de quelques affiches plus récentes visant les communautés afrocaribéennes. Se dessine alors progressivement une cartographie politique des « publics » de la lutte contre le sida. Les dizaines d'affiches que nous avons exhumées, retrouvées ou simplement considérées nous permettent ainsi de saisir, d'une manière inédite et originale, l'histoire en images (et souvent en couleurs) d'une mobilisation unique, par son ampleur, son drame, son intensité, dont elles furent des actrices à part entière. Les intégrer comme des sources de plein droit et de première main dans l'écriture de notre histoire du sida à Montréal, nous offre aussi la possibilité de rendre hommage à celles et ceux qui les ont dessinées, créées, imprimées, stockées, diffusées, affichées, collectées, sauvegardées, bref à toute une communauté qui y a trouvé un instrument de luttes certes, mais aussi un outil de mobilisation, d'affirmation et de solidarité. D'ailleurs, l'un des principaux défis qu'il nous reste à affronter dans cette recherche, et dans la mise en lumière des affiches comme archives à part entière de la lutte contre le sida qu'elle implique, est la question de la patrimonialisation de ces documents. La majorité des organismes communautaires qui ont accepté de nous ouvrir leurs portes et leurs tiroirs souhaitent en effet conserver ces traces de leurs mobilisations passées, sans nécessairement savoir où ni comment, ni d'ailleurs en avoir les moyens. Notre rôle de chercheurs engagés dans une collaboration avec ces organismes consistera donc aussi, ici, à assurer la pérennité de ces sources en facilitant les partenariats entre les différentes institutions, archivistiques d'une part et militantes de l'autre (ou les deux dans le cas des AGQ) pour que les affiches qui composent le cœur de notre étude et que nous souhaitons en partie partager au sein d'un ouvrage, puissent être sauvegardées dans des conditions permettant non seulement le travail de mémoire, mais aussi leurs questionnements futurs à l'aune d'autres perspectives, d'autres intérêts de recherche et d'autres regards.

\section{NOTES}

1. https://arquives.ca/about (consulté le 20/02/2021).

2. http://agq.qc.ca/a-propos/ (consulté le 20/02/2021).

3. En particulier celleux du Collectif archives LGBTQI : https://archiveslgbtqi.fr/ (consulté le 20/02/2021).

4. BARDOU, Florian. 2021. «Paris : la création d'un centre d'archives LGBTQI bientôt actée ?» Libération, 3 février 2021.

5. https://visualaids.org/about-us_(consulté le 20/02/2021)

6. http://www.actuporalhistory.org/_(consulté le 20/02/2021)

7. BROQUA, Christophe. 1998. "De quelques expressions collectives de la mémoire face au sida » Ethnologie française, XXVII (1) : 103-111.

8. https://www.mucem.org/page-search?term=sida (consulté le 20/02/2021). Cette collection fait l'objet d'une exposition dédiée du 15 décembre 2021 au 2 mai 2022 : https://www.mucem.org/ programme/exposition-et-temps-forts/vihsida (consulté le 20/01/2021).

9. CHANTRAINE, Renaud. 2019. «Sillonner les failles de la transmission » Vacarme, 89 : 88-93. 
10. http://lgbtqalms.co.uk/about/ (consulté le 18/05/2021).

11. Par exemple, en 2018 à Montréal : http://agq.qc.ca/les-agq-au-cinema-du-parc/ (consulté le 27/02/2021).

12. À l'exception peut-être de l'ouvrage de l'artiste militant et ancien membre du collectif Silence=Death Avram Finkelstein intitulé After Silence. A History of AIDS through Its Images (University of California Press, 2017).

13. Notamment, pour le Québec, NENGEH MENSAH, Maria. 2003. Ni vues ni connues? Femmes, VIH, médias. Montréal : Les éditions du remue-ménage.

14. https://aidsactivisthistory.ca/_(consulté le 27/02/2021).

15. BOUCHARD, Caroline et RENAUD, Lise. 2005. La santé s'affiche au Québec. Montréal : Presses de l'Université du Québec.

16. GARÇON, Jack et al. 1995. Quand la santé publique s'affiche, 50 ans, 50 affiches 1945-1995. Rennes : Éditions ENSP ; MANSOTTE, François. 2019. En pleine forme. 130 affiches pour la promotion de la santé et l'environnement. Paris : Hygée Éditions ; ou encore FRIOUX, Stéphane et NOURRISSON, Didier. 2015. Propre et sain : 100 ans d'hygiène à l'école. Paris : Armand Colin.

17. KLUSASECK, Alan et MORRISON, Ken (éds.). 1998. A leap in the dark: AIDS, art and contemporary cultures. Montreal : Vehicule Press.

18. Très majoritairement en français, parfois déclinées en français et en anglais, très rarement en espagnol ou en langue autochtone, et jamais en créole - pour les fonds que nous avons pu consulter.

19. Cette recherche s'inscrit dans le cadre d'une dynamique collective de travail sur l'histoire de la mobilisation des hommes gais face au VIH à Montréal et à Marseille, projet financé par l'Agence nationale de recherche sur le sida et les hépatites virales (numéro d'octroi : ECTZ131604 / Appel à projet 2020-2).

20. LAVOIE, René. 1998. "Deux solitudes : les organismes sida et la communauté gaie ", in Sortir de l'ombre. Histoires des communautés lesbienne et gaie de Montréal, DEMCZUK, Irène \& REMIGGI, Franck (éds.). Montréal : VLB éditeur, 337-362.

21. MORRISON, Ken. 1991. "Sero Zero : l'Histoire des interventions en milieu gai montréalais de 1985 à nos jours sur la prévention de la transmission du VIH. Où va-t-on maintenant?» in Homosexualités et sida, POLLAK, Michael, MENDES-LEITE, Rommel \& VAN DEM BORGHE, Jacques (éds.). Lille : GKC, 132-141, ici p. 135.

22. GIRARD, Gabriel et KLEIN, Alexandre. 2019. «Montréal, 1989 : la conférence qui a changé le cours de l'histoire du sida » Libération, 6 juin 2019. https://www.liberation.fr/debats/2019/06/06/ montreal-1989-la-conference-qui-a-change-le-cours-de-l-histoire-du-sida_1732079_(consulté le 03/02/2021).

23. MORRISON, Ken. 1991. « Sero Zero », op. cit., p. 137.

24. Évènement sportif LGBT organisé depuis 2006 en parallèle des Gay games - qui existent depuis 1982. La première édition des Outgames a eu lieu à Montréal.

25. GOSSELIN, Étienne. 2005. La lutte contre le sida au Québec: Le centre québécois de coordination sur le sida (1989-1995). Mémoire de maitrise en histoire, Université Sherbrooke.

26. NAMASTE, Viviane. 2019. Savoirs créoles: Leçons du sida pour l'histoire de Montréal. Montréal: Mémoires d'encrier.

27. L'un de ces documents a été retrouvé et mis en ligne dans le cadre du projet de recherche canadien AIDS activism history project: https://aidsactivisthistory.omeka.net/items/show/510 (consulté le 09/02/2021).

28. WANG, Yu. 2017. Sémiotique et rhétorique des codes socio-culturels de l'affiche et de l'affichage : le cas des

campagnes de prévention contre le SIDA. Thèse de doctorat en linguistique, Université de Lorraine. 


\section{RÉSUMÉS}

L'épidémie de VIH-sida a conduit à la production d'une multitude de documents, que ce soit par les autorités responsables de sa gestion, par les malades et leurs proches ou par les associations militantes et communautaires qui les soutenaient. Parmi ces productions, les affiches occupent une place particulière tant elles ont caractérisé - qu'on pense seulement au célèbre Silence=Death du collectif éponyme qui incarne à lui seul l'épidémie et ses enjeux politiques - à la fois la stratégie de prévention contre le VIH et les mobilisations sociopolitiques attenantes. Ces archives à part entière nous offrent ainsi une perspective unique sur le déroulement de l'épidémie, mais également sur l'implication des différents acteurs, gouvernementaux, communautaires ou associatifs, dans la lutte contre le virus. C'est ce que nous entendons démontrer dans notre projet sur l'histoire du VIH/sida à Montréal vue à travers les affiches de la lutte contre cette épidémie.

The HIV-AIDS epidemic has led to the production of a multitude of documents, whether by the authorities responsible for its management, by patients and their relatives or by the activist and community associations that supported them. Among these productions, the posters occupy a special place as they characterized - one thinks only of the famous Silence $=$ Death of the eponymous collective which alone embodies the epidemic and its political issues - both the prevention strategy against HIV and related socio-political mobilizations. These full-fledged archives thus offer a unique perspective on the course of the epidemic, but also on the involvement of different actors - governmental, community, or associative - in the fight against the virus. This is what we intend to demonstrate in our project on the history of HIV/AIDS in Montreal as seen through posters of the fight against this epidemic.

\section{INDEX}

Thèmes : Explorations

Keywords : aids, posters, Quebec, gay community, history

Mots-clés : sida, affiches, Québec, communauté gaie, histoire

\section{AUTEURS}

\section{ALEXANDRE KLEIN}

Université d'Ottawa

Alexandre Klein est philosophe et historien des sciences, spécialisé dans les questions de santé à l'époque contemporaine. Il est actuellement professeur auxiliaire à l'École des sciences infirmières de l'Université d'Ottawa au Canada et membre associé au Centre interuniversitaire de recherche sur la science et la technologie.

\section{GABRIEL GIRARD}

Inserm

Gabriel Girard est sociologue spécialisé dans les enjeux de prévention du VIH et actuellement chargé de recherche à l'Institut national sur la santé et la recherche médicale (Inserm), affilié au SESSTIM (Aix-Marseille Université - IRD - Inserm). 